\title{
Impact of Shave Biopsy on Diagnosis and Management of Cutaneous Melanoma: A Systematic Review and Meta-Analysis
}

\author{
Omid Ahmadi, BSc MBChB PGDipSurgAnat PhD ${ }^{1}$, Moushumi Das, MBChB PGDipSurgSci (Edin) ${ }^{2}$, \\ Behzad Hajarizadeh, MD MPH PhD ${ }^{3}$, and Jon A. Mathy, MD FACS FRACS ${ }^{2,4}$
}

${ }^{1}$ Department of Otolaryngology and Head and Neck Surgery, Waikato Hospital, Hamilton, New Zealand; ${ }^{2}$ Auckland Regional Plastic, Reconstructive and Hand Surgery Unit, Middlemore Hospital, Auckland, New Zealand; ${ }^{3}$ The Kirby Institute, University of New South Wales, Sydney, NSW, Australia; ${ }^{4}$ Department of Surgery, University of Auckland School of Medicine, Auckland, New Zealand

\begin{abstract}
Background. Melanoma is the most lethal skin cancer. Excision biopsy is generally recommended for clinically suspicious pigmented lesions; however, a proportion of cutaneous melanomas are diagnosed by shave biopsy. A systematic review was undertaken to investigate the impact of shave biopsy on tumor staging, treatment recommendations, and prognosis.

Methodology. The MEDLINE, Embase, and Cochrane Library databases were searched for relevant articles. Data on deep margin status on shave biopsy, tumor upstaging, and additional treatments on wide local excision (WLE), disease recurrence, and survival effect were analyzed across studies.

Results. Fourteen articles from 2010 to 2020 were included. In total, 3713 patients had melanoma diagnosed on shave biopsy. Meta-analysis revealed a positive deep margin in $42.9 \%$ of shave biopsies. Following WLE, change in tumor stage was reported in $7.7 \%$ of patients. Additional treatment was recommended for $2.3 \%$ of patients in the form of either further WLE and/or sentinel lymph node biopsy. There was high heterogeneity across studies in all outcomes. Four studies reported survival, while no studies found any significant difference in
\end{abstract}

(C) Society of Surgical Oncology 2021

First Received: 23 November 2020

Accepted: 26 February 2021;

Published Online: 29 March 2021

J. A. Mathy, MD FACS FRACS

e-mail: Jon.Mathy@middlemore.co.nz disease-free or overall survival between shave biopsy and other biopsy modalities.

Conclusions. Just over $40 \%$ of melanomas diagnosed on shave biopsy report a positive deep margin; however, this translated into a change in tumor stage or treatment recommendations in relatively few patients $(7.7 \%$ and $2.3 \%$, respectively), with no impact on local recurrence or survival among the studies analyzed.

Worldwide, more than 350,000 people are diagnosed with melanoma and nearly 60,000 deaths are attributed to melanoma annually, making it the most lethal form of skin cancer. ${ }^{1,2}$ Early diagnosis and treatment are critical to decreasing melanoma-related mortality. Tissue biopsy represents a crucial step in diagnosis. The US National Comprehensive Cancer Network (NCCN) and the European Society for Medical Oncology (ESMO) guidelines recommend narrow excision biopsy of suspicious pigmented lesions with $1-3 \mathrm{~mm}$ margins. ${ }^{3,4}$ Examining the entire lesion allows accurate measurement of Breslow thickness, which is the most significant prognostic indicator for localized disease. ${ }^{5}$ Breslow thickness also guides standard management recommendations for definitive wide local excision (WLE) and the use of sentinel lymph node biopsy (SLNB). ${ }^{6}$ In appropriately selected patients, SLNB has been shown to improve treatment outcomes in the node-positive cohort in terms of melanoma-specific survival, regional disease control, and surgical morbidity. ${ }^{7,8}$

Shave biopsy can underestimate Breslow thickness through incomplete sampling. This risks undertreatment relative to accepted standards of care with respect to both WLE margins and consideration for SLNB. On the other 
hand, advocates for shave biopsy argue that it is easier and quicker to perform, effectively lowering the threshold for early diagnosis and thereby facilitating meaningful improvement in overall treatment outcome. ${ }^{9}$

In clinical practice, patients are frequently referred to surgical oncologists for further management of melanoma that has been diagnosed on shave biopsy. In Australia and the UK, it has been reported that approximately one-quarter of patients referred to surgical oncologists were diagnosed on partial biopsy, including shave biopsy, ${ }^{10,11}$ while in the US, as many as $50 \%$ of patients referred to a tertiary treatment center are diagnosed on shave biopsy. ${ }^{12}$ Therefore, we undertook a systematic review to answer the question, "what is the impact of shave biopsy on diagnosis, management, and survival of patients with melanoma?"

\section{METHODS}

This systematic review was conducted in accordance with the Preferred Reporting Items for Systematic Reviews and Meta-Analyses statement. ${ }^{13}$ An electronic search of the Medline, EMBASE, and Cochrane Library databases was conducted using the key words 'shave biopsy', and 'melanoma'. Original human studies in the English language, published between January 2010 and November 2020, were searched. Title, abstracts, and full-text articles were screened. Studies were eligible for inclusion if they included primary data, specifically reported the number of shave biopsies in the study population and reported at least one of the following three outcomes in the shave biopsy cohort: (1) proportion of deep margin positivity; (2) proportion of T-stage change following WLE; and/or (3) proportion of treatment recommendation change following WLE. The reference list of the included studies was also manually searched for studies that met the inclusion criteria. Studies that did not meet the inclusion criteria, including lack of original data, full-text articles not published, or case reports, were excluded from the study (Fig. 1).

Data were extracted using standardized proforma. Extracted variables included study population source, number of patients who had shave biopsy, number of patients with a positive deep margin, change in $\mathrm{T}$ stage following WLE, change in treatment recommendation following WLE, and survival information, when provided.

Risk of bias was assessed using a modified scale derived from the Newcastle-Ottawa Scale for non-randomized studies (Table 1). ${ }^{14}$ This scale ranges from 0 to 5 , with points being assigned for the inclusion of patients with melanoma, regardless of stage; prospective study; reliable source of data; comment on the nature of the shave biopsy (e.g. deep shave biopsy or saucerization); and the duration of follow-up (only for studies with survival data). Studies with a score of $\leq 1,2-3$, and $>4$ were considered to have a high, moderate, and low risk of bias, respectively.

The primary outcome of this systematic review was the proportion of patients having a positive deep margin following shave biopsy, while secondary outcomes measured were the proportion of patients who had T-stage change following WLE and the proportion with a change in treatment recommendation according to the NCCN clinical practice guidelines for cutaneous melanoma. ${ }^{3}$ A positive deep margin was defined as the presence or transection of the melanoma at the deep margin of the pathological specimen during histopathological examination. The change in T-stage was defined based on the 8th edition of American Joint Committee on Cancer (AJCC) staging manual for cutaneous melanoma. ${ }^{6}$

The $I^{2}$ statistic was used to assess heterogeneity across studies. ${ }^{15}$ Meta-analysis was used to cumulate the proportions of deep margin positivity, change in $\mathrm{T}$ stage after WLE, and change in treatment recommendation after WLE. Random-effect models were used in all analyses given an $I^{2}$ of $\geq 25 \%$. All analyses were performed using Stata 14.0 (StataCorp LLC, College Station, TX, USA). Meta-synthesis was used to assess the impact of shave biopsy on overall and disease-free survival.

\section{RESULTS}

No existing systematic reviews examining the shave biopsy effect on melanoma management or outcomes were identified. A total of 14 studies, including 3713 melanomas diagnosed on shave biopsy, met the inclusion criteria and were included in the analysis. The studies varied in regard to their study population and methodology. Most studies only reported results on a specific aspect of interest (e.g. proportion of positive deep margin or proportion of change in T stage). The characteristics of the included studies have been outlined in Table 2 .

\section{Deep Margin Status}

Overall, 11 studies comprising 2846 shave biopsies reported 1259 positive deep margins. This corresponds to a combined deep margin positivity of $42.9 \%$ (95\% confidence interval $[\mathrm{CI}]$ 27.8-58.0\%), as shown in Fig. 2. Regarding the impact of lesion thickness on deep margin status, Doolan et al., in a study of 455 patients, further reported that the rate of deep margin positivity increased from $48 \%$ for those with melanomas $<1 \mathrm{~mm}$ in Breslow thickness, to $90 \%$ in those with Breslow thickness $>2 \mathrm{~mm} \cdot{ }^{26}$ High heterogeneity was observed across studies $\left(I^{2}=98.9 \%\right)$. 


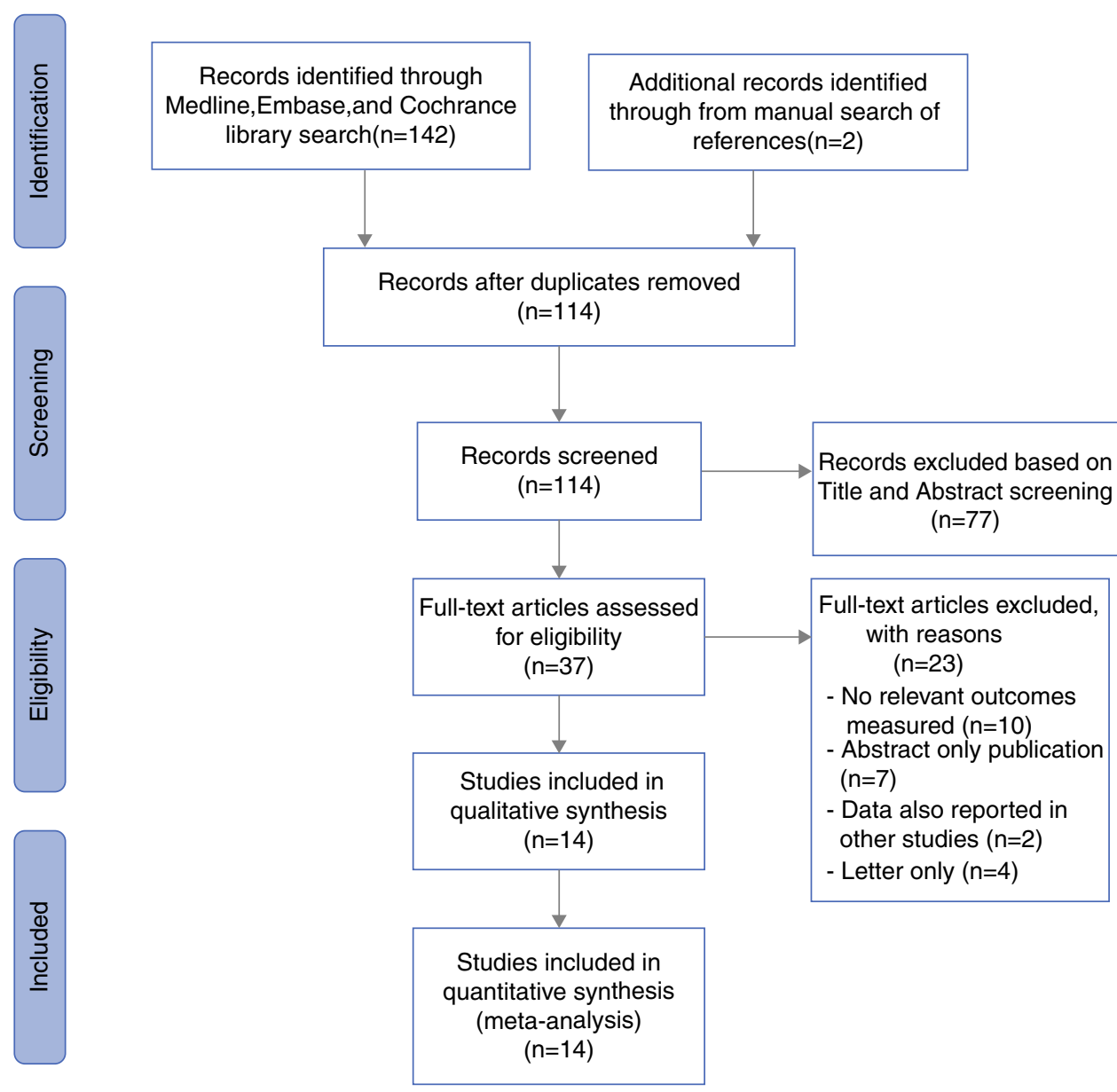

FIG. 1. Flow diagram of the review process

TABLE 1 Modified Newcastle-Ottawa quality assessment scale used to assess risk of bias among the studies included in the systematic review $^{14}$

\begin{tabular}{|c|c|c|c|}
\hline & & Point $=1$ & Point $=0$ \\
\hline \multirow[t]{2}{*}{ Selection } & $\begin{array}{l}\text { Representativeness of the study } \\
\text { population }\end{array}$ & $\begin{array}{l}\text { Patients with melanoma (regardless of } \\
\text { stage) included }\end{array}$ & $\begin{array}{l}\text { Only subset of melanoma stages included in } \\
\text { the study }\end{array}$ \\
\hline & Nature of the study & Prospective study & Retrospective study \\
\hline \multirow[t]{2}{*}{ Exposure } & Ascertainment of exposure & Secure record (e.g. surgical record) & Written self-report or no description \\
\hline & Type of shave biopsy & $\begin{array}{l}\text { Commented on 'deep shave' or standard } \\
\text { shave biopsy }\end{array}$ & No description \\
\hline Outcome & $\begin{array}{l}\text { For studies with survival data, follow- } \\
\text { up length }\end{array}$ & $\geq 5$ year follow-up & $<5$-year follow-up \\
\hline
\end{tabular}

\section{T Staging and Proportionate Change in T Stage}

Following Wide Local Excision (WLE)

The staging system utilized for determining $\mathrm{T}$ stage varied among the studies. The AJCC 6th edition was used by Hieken et al., ${ }^{17}$ the 7 th edition was used by Mills et al. ${ }^{18}$ and Saco and Thigpen, ${ }^{20}$ and the 8 th edition was used by Doolan et al. ${ }^{26}$ and De Menezes et al. ${ }^{27}$ In four studies, it was not specified which version of the AJCC was used. $^{9,12,22,25}$ T-stage upstaging following WLE was captured by eight studies $(n=1883)$. In a study of 853 patients with melanoma, Kaiser et al. have shown that those diagnosed by shave biopsy were significantly more likely to be diagnosed with a thinner melanoma than those diagnosed by excision biopsy. ${ }^{21}$ In this study, in the shave biopsy group, $77 \%$ of patients had $\mathrm{T} 1,15 \%$ had $\mathrm{T} 2$, and $7 \%$ had T3-4 melanomas, while in the excision biopsy group $57 \%$ had $\mathrm{T} 1,19 \%$ had $\mathrm{T} 2$, and $24 \%$ had T3-4 disease 


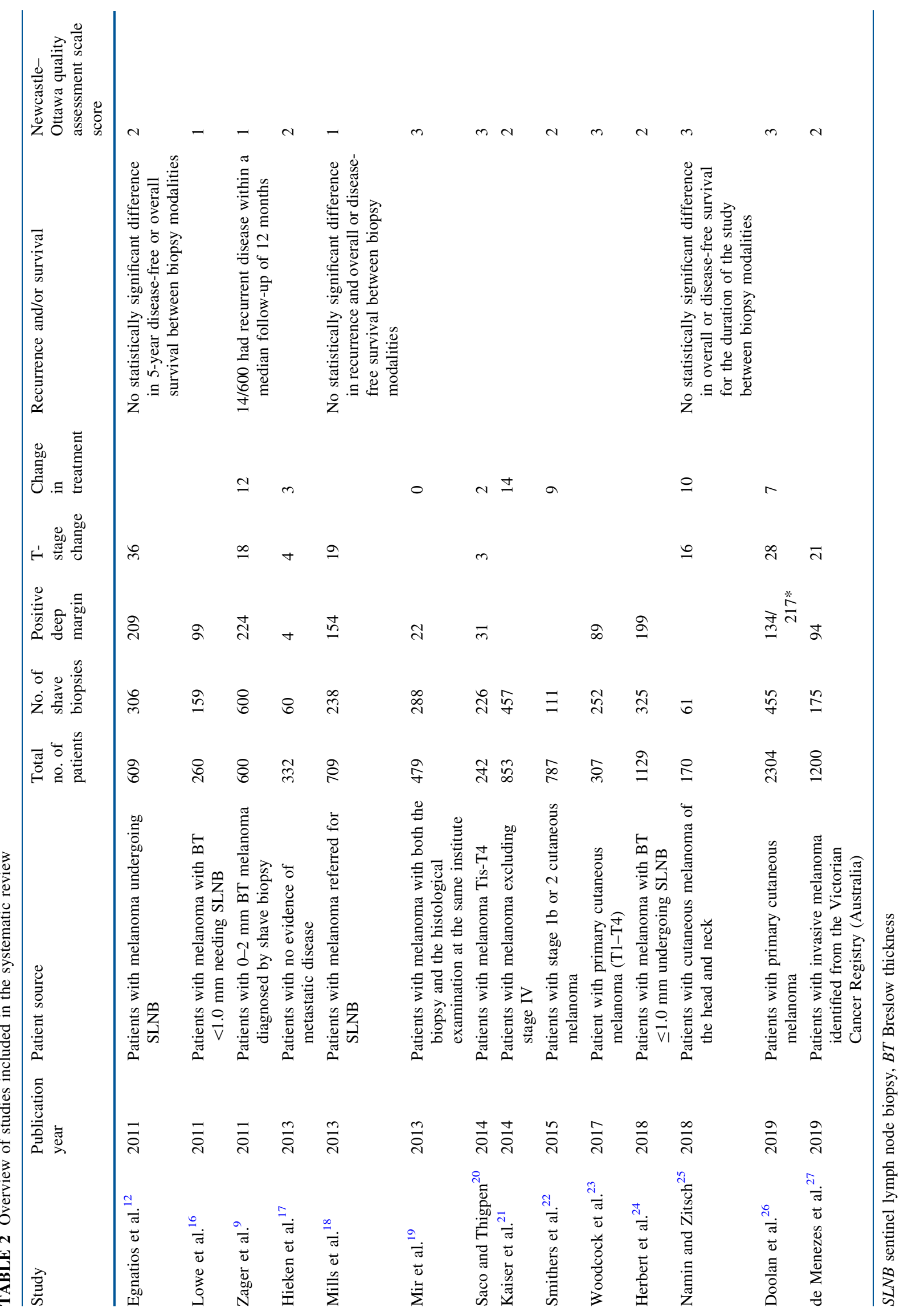


FIG. 2. Forest plot of the estimated proportion of deep margin positivity following shave biopsy of melanoma. ES effect size, $C I$ confidence interval. *data on deep margin positivity following shave biopsy was reported for 217 of the cases

Positive Deep Margin

\begin{tabular}{|c|c|c|c|}
\hline Study & & ES $(95 \% \mathrm{Cl})$ & Weight \\
\hline Egnatios, 2011[12] & 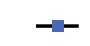 & $68.30(62.89,73.26)$ & $9.1 \%$ \\
\hline Zager, 2011[9] & & $37.33(33.56,41.27)$ & $9.2 \%$ \\
\hline Lowe, 2011[16] & 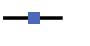 & $62.26(54.52,69.43)$ & $9.0 \%$ \\
\hline Mills, 2013[18] & $\rightarrow-$ & $64.71(58.44,70.50)$ & $9.1 \%$ \\
\hline Mir, 2013[19] & & $7.64(5.10,11.29)$ & $9.2 \%$ \\
\hline Hieken, 2013[17] & & $6.67(2.62,15.93)$ & $9.1 \%$ \\
\hline Saco, 2014[20] & & $13.72(9.83,18.81)$ & $9.1 \%$ \\
\hline Woodcock, 2017[23] & & $35.32(29.68,41.40)$ & $9.1 \%$ \\
\hline Herbert, 2018[24] & $-\square$ & $61.23(55.83,66.37)$ & $9.1 \%$ \\
\hline Doolan et al. 2019[26] $\left(134 / 217^{\star}\right)$ & 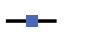 & $61.75(55.13,67.96)$ & $9.1 \%$ \\
\hline De Menezes, 2019[27] & - & $53.71(46.33,60.94)$ & $9.0 \%$ \\
\hline Overall $\left(I^{\wedge} 2=98.95 \%, p=0.00\right)$ & & $42.90(27.78,58.03)$ & $100 \%$ \\
\hline
\end{tabular}

$(p<0.001)$. de Menezes et al. reported T-stage change in 21 patients following WLE, the proportion of which increased with increasing tumor thickness. ${ }^{27}$ These included eight patients who were upstaged from T1 melanoma (6\% of total patients with T1 melanoma), eight who were upstaged from T2-3 (25\% of total patients with T2-3 melanoma), and five who had their T4 melanomas upstaged (38\% of total patients with T4 melanoma). Saco and Thigpen reported that $3 / 226$ patients who were diagnosed with melanoma on shave biopsy had their T-stage increased following WLE. ${ }^{20}$ These included one patient who had their T1 melanoma upstaged to T4, one who had their T2 melanoma upstaged to T3, and another who had their T3 melanoma upstaged to T4 melanoma. In their study of 170 head and neck cutaneous melanomas, Namin and Zitsch reported that patients diagnosed with melanoma following excision biopsy were more likely to be diagnosed with a more advanced overall stage than those diagnosed by shave biopsy; however, this difference was not statistically significant. $^{25}$ In total, 145 cases were upstaged, which corresponded to a combined proportion of T-stage upgrade following WLE of $7.7 \% \quad(95 \%$ CI 4.6-10.8\%), as shown in Fig. 3. High heterogeneity was observed across studies $\left(I^{2}=89.7 \%\right)$.

\section{Proportionate Change in Treatment Recommendation Following WLE}

Not all patients with T-stage upgrade require additional treatments according to treatment standards. The need for additional treatment following WLE was captured by eight studies $(n=2258)$, as illustrated in Fig. 4. Changes in treatment recommendation included wider WLE margin with or without SLNB. In total, 57 patients needed additional treatment following WLE, corresponding to a combined proportion of $2.3 \%$ (95\% CI $0.88-3.61 \%$ ) of patients requiring additional treatment. Specifically, among studies that provided details on the change in treatment recommendation following WLE, 21/1281 (1.6\%) patients had a change in the recommended WLE margin, and $8 / 1114(0.7 \%)$ were candidates for SLNB. Kaiser et al. found no statistically significant difference in treatment recommendation change for patients diagnosed by shave biopsy based on location between the head and neck, trunk, or extremity sites. ${ }^{21}$ High heterogeneity was observed across studies $\left(I^{2}=84.0 \%\right)$.

\section{Impact of Shave Biopsy on Recurrence and Survival}

Egnatios et al. retrospectively examined the data of patients with malignant melanoma who underwent SLNB between 1997 and 2010 at the Mayo Clinic, Rochester, MN, USA. $^{12}$ In total, 609 patients were treated, of whom 306 (50.2\%) were diagnosed based on shave biopsy. The 5-year disease-free survival was not different between the shave/ punch or excision biopsy groups $(75 \%$ vs. $77 \% ; p=0.74)$. Similarly, the 5-year overall survival was not statistically different between these groups (79\% vs. $83 \% ; p=0.44)$.

Namin and Zitsch retrospectively examined 170 patients who were diagnosed with head and neck cutaneous 
FIG. 3. Forest plot of the estimated proportion of change in T stage following WLE of melanoma diagnosed on shave biopsy. $E S$ effect size, $C I$ confidence interval, WLE wide local excision
T-stage Change After WLE

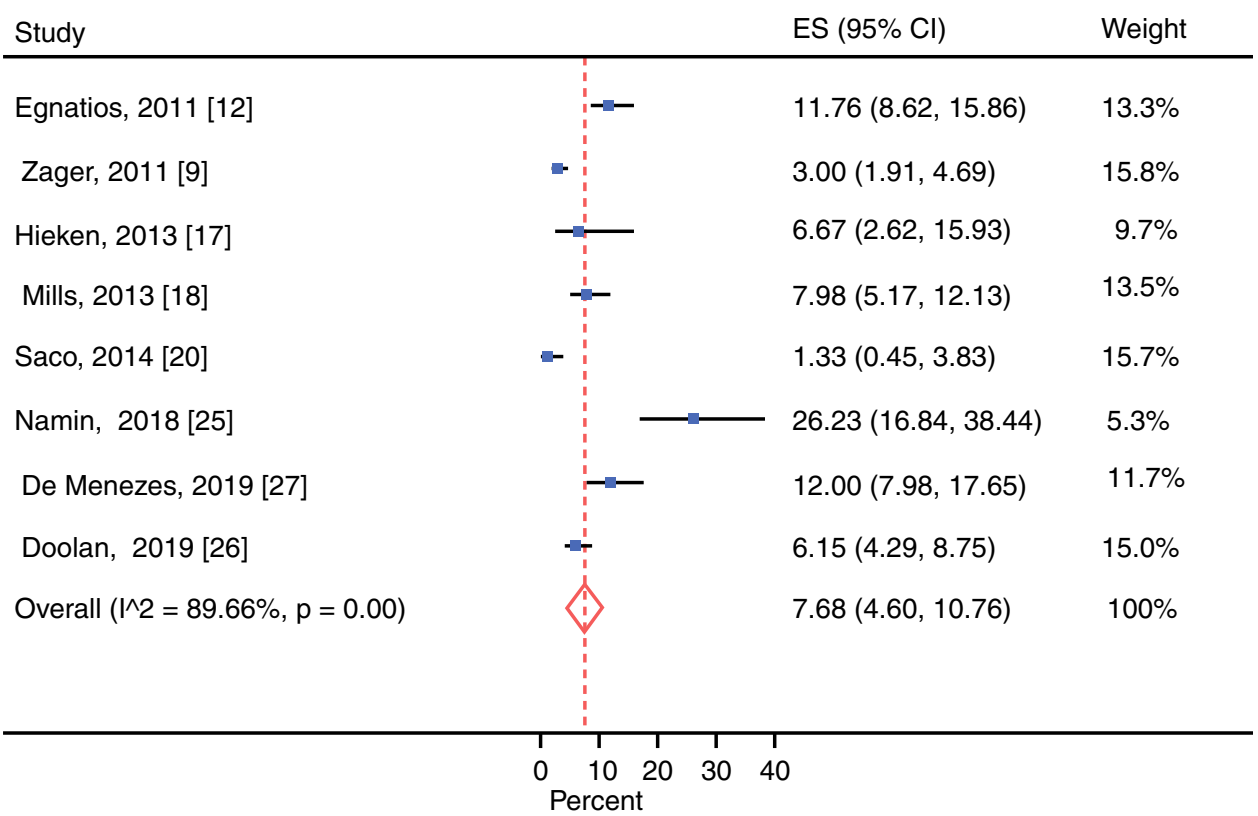

melanoma between 2000 and 2015 at the University of Missouri, Columbia, MO, USA $;{ }^{25}$ of these patients, 61 had shave biopsy. Namin and Zitsch found no significant difference in disease-free or overall survival for the duration of the study based on the biopsy modality.

In a retrospective study of 600 patients diagnosed with melanoma (Breslow thickness $<2 \mathrm{~mm}$ ) on shave biopsy between 2006 and 2009, Zager et al. established a median follow-up of 12 months. ${ }^{9}$ During the follow-up period, $14 / 600$ patients developed recurrent disease; the median Breslow thickness of melanoma in these patients was $1.67 \mathrm{~mm}$. The authors concluded that even in partial sampling, with the risk of diagnostic and T-stage inaccuracy, this does not appear to impact the long-term outcome.

Mills et al. conducted a retrospective study of 709 patients with malignant melanoma who were referred for SLNB between 1998 and 2012, with a mean follow-up time of 43 months. ${ }^{18}$ In this study, 238 (33.6\%) patients were diagnosed based on shave biopsy. The authors found that 105 patients had recurrence and 79 had died (51 from melanoma) during the course of the study; however, they found no statistically significant difference in recurrence, overall survival, or disease-specific survival between biopsy types.

\section{DISCUSSION}

To our knowledge, this is the first systematic review to examine the impact of shave biopsy on cutaneous melanoma staging, treatment, and prognosis. Cumulatively, there is a high rate of reported deep margin positivity with shave biopsy (42.9\%); however, this translated into a change in T stage following WLE in $7.7 \%$ of patients, and a change in treatment recommendation in $2.3 \%$ of patients. There was no impact on long-term recurrence and diseasefree or overall survival over a variable period of follow-up among studies.

Accurate biopsy of cutaneous melanoma is important for a number of reasons, but perhaps the most important reason is to establish the true Breslow thickness. Breslow thickness guides further treatment according to standard practice guidelines, such as the WLE margin and the role for SLNB. It is also the strongest prognostic indicator for patients with localized cutaneous melanoma. ${ }^{6,26}$ Many international and national guidelines recommend excision biopsy as the preferred mode for biopsy of suspicious pigmented skin lesions because it allows for examination of the entire lesion and therefore provides the most accurate measurement of true Breslow thickness. ${ }^{3,28-30}$ Additional treatments following a melanoma diagnosis include WLE and SLNB. ${ }^{7,8}$

While excision biopsy represents the standard of care in the initial evaluation of patients with suspicious cutaneous lesions, it is not without its drawbacks. Relative to shave biopsy excision, biopsy generally requires access to more procedure space, more procedure time, more equipment, more advanced technical skills such as suture wound closure, higher expense, and, in some cases, arguably leaves a more visible scar. These requirements can be particularly influential in selecting a threshold for subjecting patients to excision biopsy where suspicion of cutaneous melanoma is low, when the lesion is located in an aesthetically sensitive 
FIG. 4. Forest plot of the estimated proportion of change in treatment recommendation following WLE of melanoma diagnosed on shave biopsy. ES effect size, $C I$ confidence interval, WLE wide local excision
Patients needing additional treatment after WLE

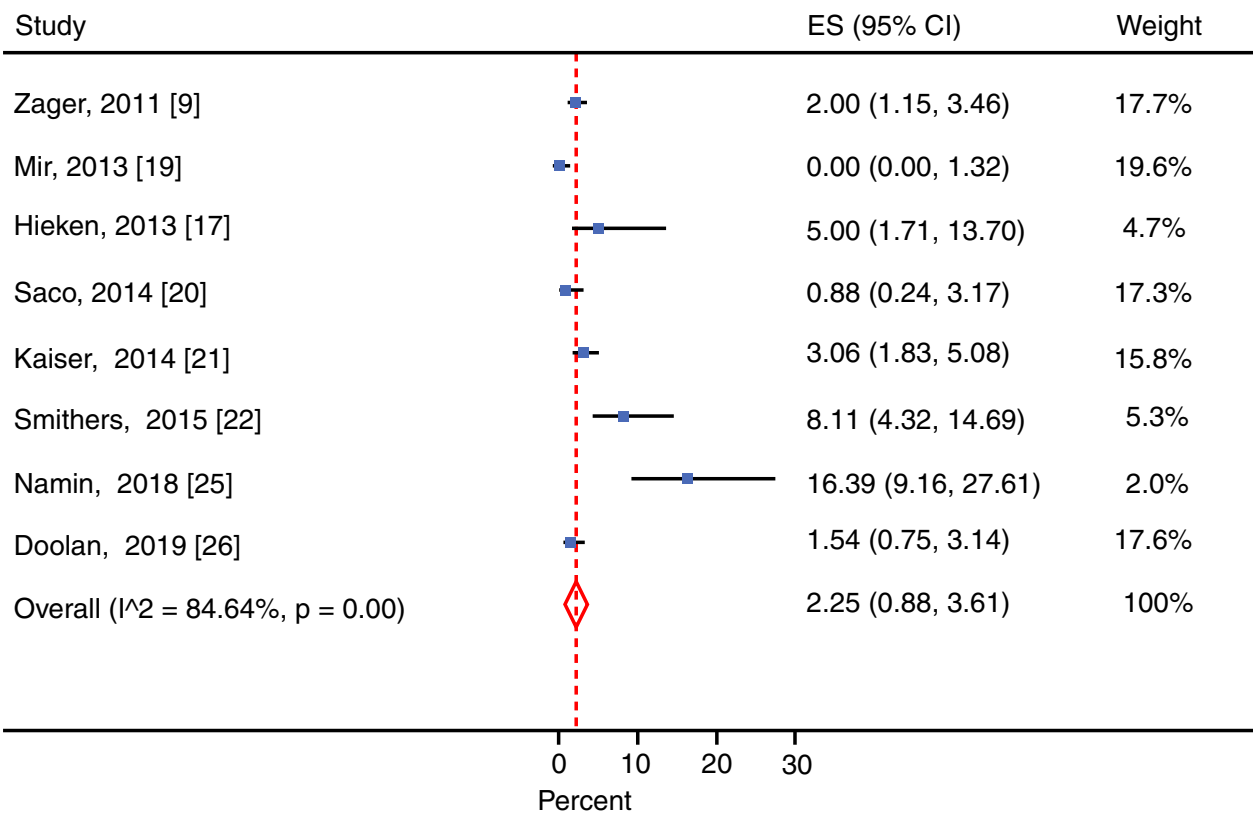

area, and in under-resourced patient and clinical environments and/or time-sensitive environments. The drawbacks are particularly notable for first-line practitioners such as community doctors and general practitioners, as well as those working in high-volume or resource-restrained clinics where serial examination or immediate biopsy is not feasible. As a result, biopsy of atypical lesions could be deferred, or patients could experience additional diagnostic delays while awaiting referral to another healthcare professional for the purposes of excision biopsy. The recent coronavirus disease 2019 (COVID-19) pandemic may further result in a delay in diagnosis as a higher rate of nonattendance at specialist appointments has been observed, which has been attributed to the fear of contracting COVID-19. ${ }^{31}$ Each of these steps could result in a clinically significant delay in diagnosis and treatment. This delay could also represent a significant stressor for patients.

Worldwide, the rate of shave biopsy seems to be increasing. ${ }^{27}$ The advocates for shave biopsy argue that shave biopsy is quick, relatively cheap, requires less technical skill and equipment, and can be performed in a clinic setting without the need to refer or reschedule patients. It can also achieve a relatively favorable cosmetic result. ${ }^{19}$ These factors may reduce the barriers to biopsy of suspicious skin lesions, with some arguing any biopsy is better than no biopsy. This will likely reduce the risk of missing a melanoma in a patient with a low index who may have otherwise been scheduled for serial examination and a delay in making the diagnosis. ${ }^{27}$ A diagnosis of melanoma based on tissue biopsy, even if it is a partial biopsy, is also likely to expedite patient treatment. In a survey of family doctors in Ontario, up to $24 \%$ reported a significant delay of $>6$ months between the specialist appointment and diagnosis when patients were referred without a tissue biopsy. ${ }^{32}$

Shave biopsy is particularly popular among dermatologists, who often see a large number of high-risk patients in an outpatient clinic setting. ${ }^{21}$ Among the studies that reported the detail of the health professional providing the shave biopsy, $1609(83.1 \%)$ were performed by dermatologists, $98(5.1 \%)$ were performed by surgeons, and 230 $(11.9 \%)$ were performed by 'other' health professionals. $^{9,12,19,21,22,27}$ The level of experience between practitioners was not reported; however, Doolan et al. reported no statistically significant difference in the rate of T-stage change between different health providers following WLE. ${ }^{26}$ Similarly, Kaiser et al. reported no statistically significant difference in the rate of change in treatment recommendation following WLE between different health professionals. ${ }^{21}$ Because practitioner experience is likely to significantly impact accurate sampling by shave biopsy, further study into this, as well as effective training strategies, is warranted.

The risk of deep margin positivity with shave biopsy is significantly higher than excision biopsy, as demonstrated in this systematic review. Sampling accuracy of a shave biopsy has been shown to improve with deep shave or saucerization, with a reduced proportion of deep margin positivity; ${ }^{19}$ however, partial biopsies have their drawbacks.

Understaging a thicker lesion as T1a with a positive deep margin represents one of the greatest risks to management decisions. The risk of performing WLE without 
SLNB in a patient with T1a who also has a positive deep margin can be confounding if the subsequent upstaging of the patient so that SLNB is recommended (e.g. from Tis or T1a to a more advanced $\mathrm{T}$ stage).

The data detailed in this systematic review indicate that this scenario is relatively rare, ${ }^{20,27}$ presumably because shave biopsy is usually adequate to diagnose at least $\mathrm{T} 1 \mathrm{~b}$ or thicker lesions. Relatedly, Zager et al. examined 600 patients diagnosed with $0-2 \mathrm{~mm}$ Breslow thickness cutaneous melanomas on shave biopsy and found 224 (37.3\%) had a positive deep margin. ${ }^{9}$ Following WLE, tumor upstaging occurred in $18(3.0 \%)$ patients. This led to a recommendation for wider excision in 12 patients $(2.0 \%)$ and recommendation for SLNB in 8 patients (1.3\%). Nonetheless, it is important for surgical oncologists to be aware of this risk since it introduces management discussion about whether to repeat (completion) excision biopsy with a narrow margin or to proceed directly to WLE with or without SLNB. While it is possible to perform SLNB in a delayed fashion, accuracy and therapeutic value may be reduced following wide excision and/or local tissue rearrangement of the primary site. ${ }^{33,34}$ Inaccurate or delayed SLNB would be expected to lead to a higher risk of subsequent nodal recurrence, with potential loss of regional disease control, which represents one of the traditional reservations of surgical oncologists towards shave biopsy.

In this analysis, we found no significant difference in the rate of recurrence or survival between shave biopsy and other biopsy modalities. The data presented support the notion that shave biopsy is usually adequate to diagnose at least $\mathrm{T} 1 \mathrm{~b}$ or thicker lesions, and therefore the overall management of regional lymph node basins and thus melanoma-specific survival is unchanged. In their metaanalysis, Shellenberger et al. compared punch biopsy with excision biopsy ${ }^{35}$ and found no significant difference in Breslow thickness, melanoma recurrence, or melanomaspecific mortality. They reported a higher rate of all-cause mortality among the punch biopsy group (RR 1.5; $p=0.02$ ); however, the authors noted that two of the three studies included in their meta-analysis were biased towards older patients in the punch biopsy group, which the authors felt may explain this finding.

This systematic review has a number of limitations. The majority of the included studies were retrospective, and different studies also examined patients with different stages of melanoma. The risk of deep margin positivity is lower in thinner melanoma, therefore studies that selected patients with greater Breslow thickness melanomas are likely to overestimate the true deep margin positivity rate of melanoma with shave biopsy. ${ }^{12,18}$ There was also variability among the studies in the methodology and the results reported. These may explain the high heterogeneity observed in this systematic review. By focusing on more contemporary studies (2010 onwards), we have excluded studies that may have added more power to this systematic review. Similarly, by only including English-language studies, we may have excluded studies that should have otherwise been included in this systematic review.

\section{CONCLUSIONS}

Excision biopsy remains the gold standard of care for accurate biopsy of suspicious skin lesions for melanoma. Shave biopsy has a high rate of deep margin positivity and can complicate the management of patients who are subsequently diagnosed with cutaneous melanoma. However, a systematic review of the available data suggests that this translates to a relatively small number of patients who are upstaged, and an even smaller number who have a change in their treatment recommendation following WLE, with no reported impact on disease recurrence or survival relative to patients whose melanoma is diagnosed by excision biopsy.

FUNDING No sources of funding were used to assist in the preparation of this study.

DISCLOSURE Omid Ahmadi, Moushumi Das, Behzad Hajarizadeh, and Jon A. Mathy have no conflicts of interest to declare.

\section{REFERENCES}

1. Karimkhani C, Green AC, Nijsten T, et al. The global burden of melanoma: results from the Global Burden of Disease Study 2015. Br J Dermatol. 2017;177(1):134-40.

2. Eggermont AM, Spatz A, Robert C. Cutaneous melanoma. Lancet. 2014;383(9919):816-27.

3. National Comprehensive Cancer Network. NCCN Clinical Practice Guidelines in Oncology. Melanoma. Version 3.2018. Washington: National Comprehensive Cancer Network; 2018.

4. Dummer R, Hauschild A, Lindenblatt N, Pentheroudakis G, Keilholz U, ESMO Guidelines Committee. Cutaneous melanoma: ESMO Clinical Practice Guidelines for diagnosis, treatment and follow-up. Ann Oncol. 2015;26(Suppl 5):v126-32.

5. Kibbi N, Kluger H, Choi JN. Melanoma: clinical presentations. Cancer Treat Res. 2016;167:107-29.

6. Gershenwald JE, Scolyer RA, Hess KR, et al. Melanoma staging: evidence-based changes in the American Joint Committee on Cancer eighth edition cancer staging manual. CA Cancer J Clin. 2017;67(6):472-92.

7. Morton DL, Thompson JF, Cochran AJ, et al. Sentinel-node biopsy or nodal observation in melanoma. $N$ Engl $\mathrm{J}$ Med. 2006;355(13):1307-17.

8. Faries MB, Thompson JF, Cochran AJ, et al. Completion dissection or observation for sentinel-node metastasis in Melanoma. $N$ Engl J Med. 2017;376(23):2211-22.

9. Zager JS, Hochwald SN, Marzban SS, et al. Shave biopsy is a safe and accurate method for the initial evaluation of melanoma. J Am Coll Surg. 2011;212(4):454-60. 
10. Tadiparthi S, Panchani S, Iqbal A. Biopsy for malignant melanoma: are we following the guidelines? Ann R Coll Surg Engl. 2008;90(4):322-5.

11. Kelly JW, Henderson MA, Thursfield VJ, Slavin J, Ainslie J, Giles GG. The management of primary cutaneous melanoma in Victoria in 1996 and 2000. Med J Aust. 2007;187(9):511-4.

12. Egnatios GL, Dueck AC, Macdonald JB, et al. The impact of biopsy technique on upstaging, residual disease, and outcome in cutaneous melanoma. AM J Surg. 2011;202(6):771-7.

13. Moher D, Liberati A, Tetzlaff J, Altman DG. Preferred reporting items for systematic reviews and meta-analyses: the PRISMA statement. BMJ. 2009;339:b2535.

14. Stang A. Critical evaluation of the Newcastle-Ottawa scale for the assessment of the quality of nonrandomized studies in metaanalyses. Eur J Epidemiol. 2010;25(9):603-5.

15. Higgins JPT, Thompson SG, Deek JJ, Altman DG. Measuring inconsistency in meta-analyses. BMJ. 2003;327(7414):557-60.

16. Lowe M, Hill N, Page A, Chen S, Delman KA. The impact of shave biopsy on the management of patients with thin melanomas. Am Surg. 2011;77(8):1050-3.

17. Hieken TJ, Hernández-Irizarry R, Boll JM, Jones Coleman JE. Accuracy of diagnostic biopsy for cutaneous melanoma: implications for surgical oncologists. Int $J$ Surg Oncol. 2013;2013:196493.

18. Mills JK, White I, Diggs B, Fortino J, Vetto JT. Effect of biopsy type on outcomes in the treatment of primary cutaneous melanoma. Am J Surg. 2013;205(5):585-90.

19. Mir M, Chan CS, Khan F, Krishnan B, Orengo I, Rosen T. The rate of melanoma transection with various biopsy techniques and the influence of tumor transection on patient survival. J Am Acad Dermatol. 2013;68(3):452-8.

20. Saco M, Thigpen J. A retrospective comparison between preoperative and postoperative Breslow depth in primary cutaneous melanoma: how preoperative shave biopsies affect surgical management. J Drugs Dermatol. 2014;13(5):531-6.

21. Kaiser S, Vassell R, Pinckney RG, Holmes TE, James TA. Clinical impact of biopsy method on the quality of surgical management in melanoma. J Surg Oncol. 2014;109(8):775-9.

22. Smithers BM, Hughes MC, Beesley VL, et al. Prospective study of patterns of surgical management in adults with primary cutaneous melanoma at high risk of spread, in Queensland, Australia. J Surg Oncol. 2015;112(4):359-65.

23. Woodcock JL, Eyre ZW, Stoddard GJ, Duffin KC, Bowen AR. Clinical and pathologic factors associated with deep transection of biopsies of invasive melanoma. J Am Acad Dermatol. 2017;77(4):766-8.
24. Herbert G, Karakousis GC, Bartlett EK, et al. Transected thin melanoma: implications for sentinel lymph node staging. J Surg Oncol. 2018;117(4):567-71.

25. Namin AW, Zitsch RP. Impact of Biopsy Modality on the Management of Cutaneous Melanoma of the Head and Neck. Otolaryngol Head Neck Surg. 2018;158(3):473-8.

26. Doolan BJ, Robinson AJ, Wolfe R, et al. Accuracy of partial biopsies in the management of cutaneous melanoma. Australas $J$ Dermatol. 2019;60(3):209-13.

27. de Menezes SL, Kelly JW, Wolfe R, Farrugia H, Mar VJ. The increasing use of shave biopsy for diagnosing invasive melanoma in Australia. Med J Aust. 2019;211(5):213-8.

28. Michielin O, van Akkooi ACJ, Ascierto PA, Dummer R, Keilholz U, ESMO Guidelines Committee. Cutaneous melanoma: ESMO Clinical Practice Guidelines for diagnosis, treatment and followup. Ann Oncol. 2019;30(12):1884-901.

29. Cancer Council Australia Melanoma Guidelines Working Party. Clinical practice guidelines for the diagnosis and management of melanoma. Sydney: Cancer Council Australia. https://wiki.cance r.org.au/australia/Guidelines:Melanoma. Accessed 3 Nov 2020

30. Australian Cancer Network Melanoma Guidelines Revision Working Party. Clinical practice guidelines for the management of melanoma in Australia and New Zealand. Wellington: New Zealand Guidelines Group 30. The Cancer Council Australia and Australian Cancer Network; 2008

31. Fung THM, Kuet ML, Patel MK, Puri P. Addressing COVID-19 fear to improve clinic attendance for patients with wet age-related macular degeneration. Acta Ophthalmol. 2012;99(2):e285. http s://doi.org/10.1111/aos.14520.

32. Lutz K, Hayward V, Joseph M, Wong E, Temple-Oberle C. Current biopsy practices for suspected melanoma: a survey of family physicians in Southwestern Ontario. Plast Surg (Oakv). 2014;22(3):175-8.

33. Perissinotti A, Rietbergen DD, Vidal-Sicart S, Riera AA, Olmos RAV. Melanoma and nuclear medicine: new insights and advances. Melanoma Manag. 2018;5(1):MMT06.

34. Uren RF, Howman-Giles R, Thompson JF. Patterns of lymphatic drainage from the skin in patients with melanoma. $J$ Nucl Med. 2003;44(4):570-82.

35. Shellenberger RA, Fayyaz F, Sako Z, et al. Impact of biopsy technique on clinically important outcomes for cutaneous Melanoma: a systematic review and meta-analysis. Mayo Clin Proc Innov Qual Outcomes. 2020;4(4):373-83.

Publisher's Note Springer Nature remains neutral with regard to jurisdictional claims in published maps and institutional affiliations. 